\title{
PARÁMETROS GENÉTICOS DE LAS POBLACIONES OVINAS DE LA COMUNIDAD AUTÓNOMA DE CANTABRIA
}

\author{
POPULATION GENETIC PARAMETERS OF THE SHEEP POPULATIONS FROM \\ THE COMUNIDAD AUTÓNOMA DE CANTABRIA
}

\author{
Méndez, S. ${ }^{1}$, Dunner, S. ${ }^{1}$, Sañudo, B. ${ }^{2}$, García, J.A. ${ }^{2}$, Argüello, S. de ${ }^{2}$, Barquín, F. ${ }^{2}$, \\ Chomón, N. ${ }^{2}$, Calderón, L.A. ${ }^{2}$ y Cañón, J. ${ }^{\text {** }}$
}

1Departamento de Producción Animal. Facultad de Veterinaria. 28040 Madrid. España. *jcanon@vet.ucm.es ${ }^{2}$ Consejería de Desarrollo Rural, Ganadería, Pesca y Biodiversidad. Santander. España.

\section{Palabras clave adicionales}

Diversidad genética. Microsatélite.

\section{RESUMEN}

Se caracterizaron genéticamente dos poblaciones ovinas, Guriezo y Blanca de la Peña, presentes en la C.A. de Cantabria y se analizaron las relaciones con otras razas afines geográfica o culturalmente. Para ello se genotiparon un total de 21 marcadores de tipo microsatélite en 457 muestras pertenecientes a las dos razas locales cántabras, y a las razas Lacha, Bardhoka, Anogeiano, Gentile di Puglia, Laticauda, Churra Bragancana, Colmenareña, Manchega, Merina, Rubia del Molar y Segureña. Los resultados indicaron la proximidad genética esperada entre la oveja Lacha y la población de Guriezo, y la diferenciación de las dos poblaciones cántabras (tanto Guriezo como Blanca de la Peña) con respecto a las demás razas ovinas estudiadas.

\section{SUMMARY}

Two sheep populations present in the Cantabria region in the North of Spain and named Guriezo and Blanca de la Peña were genetically characterised, also their origin and relationships with other geographically or culturally connected breeds were studied. A total of 457 individuals belonging to the two groups and to other such as Lacha, Bardhoka, Anogeiano, Gentile di Puglia, Laticauda, Churra Bragancana, Colmenareña, Manchega, Merina, Rubia del Molar and Segureña breeds were genotyped for 21 microsatellite markers. The results allow detecting the influence

Presentado al Congreso SERGA (2010, Asturias).

\section{Additional KeYWoRds}

Genetic diversity. Microsatellite.

of the Lacha breed on the Guriezo population, but also the genetic differences of both sheep populations (Guriezo and Blanca de la Peña) from all others ovine breeds studied.

\section{INTRODUCCIÓN}

En la Comunidad Autónoma de Cantabria se explotan dos poblaciones ovinas (Blanca de la Peña, y de Guriezo) geográficamente distantes, cuyo origen en ambos casos podría ser el tronco Churro junto con las razas Vascas Lacha y Churra. La primera de ellas se localiza en el SW de Cantabria, en el valle de Liébana (municipios de Cillorigo y Tresviso), en el que la oveja Blanca de la Peña parece bien adaptada a la orografía y condiciones climáticas de la zona, permitiendo su explotación. Esta población ovina incluye animales eumétricos, de proporciones sublonguilíneas, perfil subconvexo, cabeza mediana con cuernos tanto en los machos como en las hembras, y de lana basta que se transforma en pelo en la zona de la garganta, región ventral y umbilical. En general la población se orienta a la producción láctea siendo destinada la leche al igual que la de cabra y vaca, con las que se mezcla, a la producción de quesos artesanales, Quesucos de Liébana y Picon 


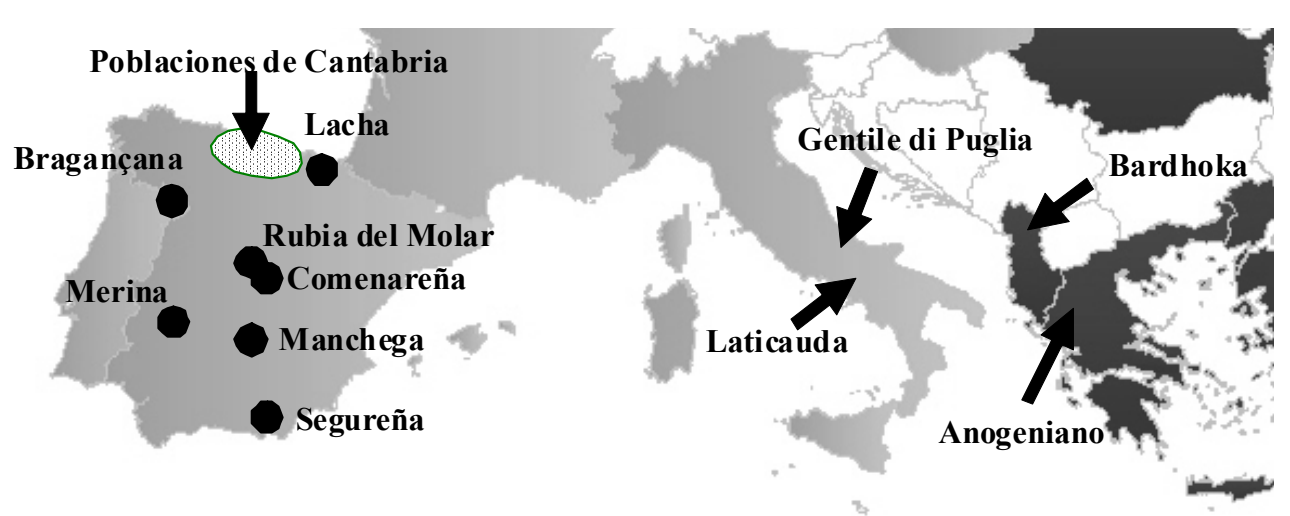

Figura 1. Posición geográfica aproximada de las poblaciones y razas ovinas incluidas en el análisis. (Geographic position of the populations and breeds included into the analysis).

Bejes-Tresviso, ambos con Denominación de Origen reconocida (B.O.E. 21/3/1994 y B.O.E. 8/3/94).

La oveja de Guriezo se localiza en la comarca oriental de la comunidad autónoma, en los municipios de Guriezo y Castro Urdiales, ambos limítrofes con la provincia de Vizcaya, concretamente con la comarca de las Encartaciones. Esta ubicación geográfica determina que se trate de una población que, independientemente de su origen ancestral, actualmente parece el resultado del cruce entre las poblaciones Carranzana y Lacha, de tal modo que la mayoría de los individuos responden fielmente al estándar racial de una de las razas mencionadas. Es

Tabla I. Número de animales analizados $(n)$, diversidad génica $\left(H_{e}\right)$, heterocigosis observada $\left(H_{o}\right)$, número efectivos de alelos $(N E A)$, nivel de consanguinidad $\left(F_{\text {IS }}\right)$ y distancia genética $\left(F_{S T}\right)$ de cada raza al resto. (Number of samples per breed $(\mathrm{n})$, gene diversity $\left(\mathrm{H}_{\mathrm{e}}\right)$, observed heterozygosity $\left(\mathrm{H}_{\mathrm{o}}\right)$, effective number of alleles (NEA), inbreeding level $\left(\mathrm{F}_{\mathrm{IS}}\right)$, and genetic distances $\left(\mathrm{F}_{\mathrm{ST}}\right)$ from each breed to the rest of breeds).

\begin{tabular}{lcccccc}
\hline Raza & $\mathrm{n}$ & $\mathrm{H}_{\mathrm{e}}$ & $\mathrm{H}_{\mathrm{o}}$ & $\mathrm{NEA}$ & $\mathrm{F}_{\text {IS }}$ & $\mathrm{F}_{\text {ST }}$ \\
\hline Guriezo & 44 & 0,694 & 0,675 & 3,3 & $0,027^{\mathrm{NS}}$ & 0,049 \\
Blanca de la Peña & 75 & 0,658 & 0,642 & 3,0 & $0,024^{\mathrm{NS}}$ & 0,055 \\
Lacha & 30 & 0,695 & 0,676 & 3,3 & $0,027^{\mathrm{NS}}$ & 0,049 \\
Colmenareña & 31 & 0,661 & 0,588 & 3,0 & 0,113 & 0,060 \\
Manchega & 31 & 0,707 & 0,663 & 3,4 & 0,063 & 0,052 \\
Merina & 31 & 0,738 & 0,679 & 3,8 & 0,081 & 0,031 \\
Rubia del Molar & 31 & 0,681 & 0,641 & 3,1 & 0,060 & 0,077 \\
Segureña & 31 & 0,734 & 0,679 & 3,8 & 0,076 & 0,028 \\
Anogeiano & 31 & 0,711 & 0,668 & 3,5 & 0,062 & 0,048 \\
Gentile di Puglia & 31 & 0,727 & 0,673 & 3,7 & 0,075 & 0,036 \\
Laticauda & 31 & 0,737 & 0,683 & 3,8 & 0,074 & 0,048 \\
Churra Bragancana & 30 & 0,728 & 0,645 & 3,7 & 0,115 & 0,038 \\
Bardhoka & 31 & 0,748 & 0,690 & 4,0 & 0,079 & 0,045 \\
\hline
\end{tabular}

NSNo significativo; NEA: Número efectivo de alelos. 


\section{CARACTERIZACIÓN GENÉTICA DE OVINOS DE CANTABRIA}

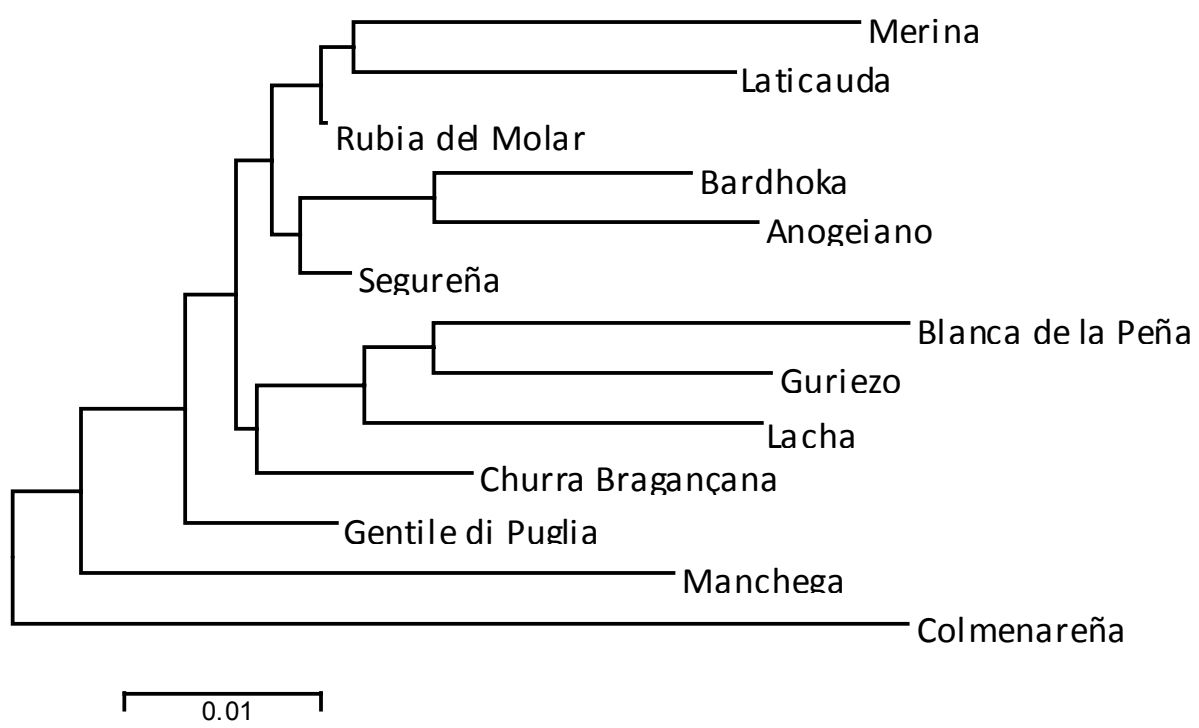

Figura 2. Representación gráfica mediante el algoritmo Neigbour-joining de las distancias genéticas $\left(F_{S T}\right)$ entre pares de razas. (Dendrogram using the $\mathrm{F}_{\mathrm{ST}}$ genetic distances among the sheep breeds).

una población de aptitud lechera con producciones superiores a 70 litros con una lactación de 5-6 meses. En la actualidad existe una asociación de criadores que engloba a 17 ganaderos con un censo aproximado de 2000 cabezas.

Con el fin de tomar decisiones sobre la necesidad del establecimiento de algún programa de conservación de estas poblaciones, parece importante una caracterización genética mínima, lo que constituyó el objeto de este trabajo.

\section{MATERIAL Y MÉTODOS}

Se utilizaron 21 marcadores de tipo microsatélite para genotipar 44 muestras de sangre de ovejas de la población de Guriezo y 75 de la población Blanca de la Peña.

Se analizaron además 338 muestras de ovejas de las razas españolas Lacha, Colmenareña, Manchega, Merina, Rubia del Molar y Segureña, italianas Anogeiano, Gentile di Puglia, Laticauda, portuguesa Churra Bragançana y la egipcia Bardhoka (figura 1). Se calcularon los principales estadísticos poblacionales mediante la macro de Excel MICROSATELLITE TOOLKIT (Park 2001), los estadísticos de Wright mediante el programa GENETIX 4.0 (Belkhir and Borsu, 1998), y se asignó el genoma de los individuos a un número de orígenes previamente determinado mediante el software STRUCTURE (Pritchard etal.,2000).

\section{RESULTADOSYDISCUSIÓN}

En la tabla I se presentan los resultados de diversidad génica, heterocigosis observada y el número efectivo de alelos, así como el nivel de consanguinidad $\mathrm{F}_{\mathrm{IS}}$, y la distancia genética $\mathrm{F}_{\mathrm{ST}}$ de cada una de las razas con respecto al resto. Podemos observar que los niveles de consanguinidad $\left(\mathrm{F}_{\mathrm{IS}}\right)$ para la población Blanca de la Peña, Guriezo y Lacha no fueron significativos, y la distancia media al resto de poblaciones $\left(\mathrm{F}_{\mathrm{ST}}\right.$ medio 0,05$)$ indicó un nivel significativo de aislamiento reproductivo. 
MÉNDEZ ET AL.

Tabla II. Porcentaje de cada población ovina que se asigna a cada uno de los cinco grupos genéticos que a priori se han considerado. (Estimated memberships fraction of sheep breeds for each of the five inferred clusters).

\begin{tabular}{llcccr}
\hline \multicolumn{5}{c}{ Grupos genéticos } \\
\hline Blanca de la Peña & $\mathbf{0 , 7 2 1}$ & $\mathbf{0 , 1 9 4}$ & 0,023 & 0,035 & 0,029 \\
Guriezo & 0,068 & $\mathbf{0 , 8 4 3}$ & 0,021 & 0,051 & 0,018 \\
Lacha & 0,056 & $\mathbf{0 , 7 6 3}$ & 0,054 & $\mathbf{0 , 1 0 9}$ & 0,018 \\
Bardhoka & 0,033 & 0,036 & $\mathbf{0 , 8 2 7}$ & 0,055 & 0,049 \\
Anogeiano & 0,033 & 0,022 & $\mathbf{0 , 7 9 8}$ & $\mathbf{0 , 1 3 1}$ & 0,015 \\
Gentile di Puglia & 0,032 & 0,071 & $\mathbf{0 , 2 8 1}$ & $\mathbf{0 , 5 8 2}$ & 0,034 \\
Laticauda & 0,025 & 0,052 & $\mathbf{0 , 1 4 6}$ & $\mathbf{0 , 7 3 9}$ & 0,038 \\
Churra Bragançana & 0,046 & 0,102 & $\mathbf{0 , 1 5 7}$ & $\mathbf{0 , 6 5 3}$ & 0,043 \\
Manchega & 0,026 & 0,062 & $\mathbf{0 , 1 0 5}$ & $\mathbf{0 , 2 5 9}$ & 0,548 \\
Merina & 0,065 & 0,105 & $\mathbf{0 , 1 7 3}$ & $\mathbf{0 , 6 3 2}$ & 0,025 \\
Segureña & 0,038 & 0,130 & $\mathbf{0 , 4 6 6}$ & $\mathbf{0 , 3 2 0}$ & 0,046 \\
Colmenareña & 0,047 & 0,035 & 0,050 & 0,079 & $\mathbf{0 , 7 9 0}$ \\
Rubia del Molar & 0,067 & 0,073 & $\mathbf{0 , 2 2 0}$ & $\mathbf{0 , 5 5 9}$ & 0,080 \\
\hline
\end{tabular}

La figura 2 presenta un dendrograma basado en la distancia $\mathrm{F}_{\mathrm{ST}}$, $\mathrm{y}$ se puede observar la agrupación de las poblaciones cántabras Blanca de la Peña y Guriezo, con la raza Lacha.

El reducido número de grupos genéticos, cinco, que es necesario considerar para que las poblaciones de Cantabria se separen del resto de razas ovinas, exceptuando la Lacha, muestran, otra vez, su grado de aislamiento reproductivo. La población Blanca de la Peña se separa claramente de la otra población ovina de Cantabria, aunque se observa que en un $20 \%$ aproximadamente comparte el mismo origen genético, que es también el de la Lacha (tabla II). Este hecho

\section{BIBLIOGRAFIA}

Belkhir, K., Borsa, P., Chikhi, L., Raufaste, N. and Bonhomme, F. 2001. Genetix, logiciel sous Windows TM pour la génétique des populations, laboratoire génome, populations, interactions. CNRS UPR 9060. Université de Montpellier II. Montpellier. France. Available online: http:// www.genetix.univ-montp2.fr/genetix/intro.htm. Park, S.D.E. 2001. Trypanotolerance in West es muy posible la consecuencia de la introducción de algún macho de ese origen, y cuando se considera un número de orígenes genéticos próximo al que proporciona la máxima verosimilitud de los datos $(\sim 12$, datos no presentados) parece indicar claramente que esa introgresión sería originaria de la población de Guriezo, y menos probablemente de origen Lacho.

En resumen, se puede señalar el origen, en gran parte, común de la raza Lacha y la población ovina de Guriezo, y una clara diferenciación de la población ovina Blanca de la Peña respecto al resto de razas consideradas en el análisis, incluidas la de Guriezo y Lacha.
African cattle and the population genetic effects of selection. Ph.D. Thesis. University of Dublin. Dublin. Ireland.

Pritchard J.K., Stephens, M. and Donnelly, P. 2000. Inference of population structure from multilocus genotype data. Genetics, 155: 945-59. Available online: http://pritch.bsd.uchicago.edu/ structure.html.

Archivos de zootecnia vol. 60, núm. 231, p. 424. 